erlebbares Abbild. Zur 25-jährigen Jubiläumsveranstaltung bleibe die INTERGEO ihrem Weg treu: „Wir setzen auf Kontinuität und Evolution. Das erwarten unsere Kunden. Daran wollen wir uns messen.“

Die INTERGE0 2019 findet statt (Pressemitteilung vom 17. bis 19. September 2019 gis-report-news)

\section{- GIS Talk 2018}

\section{"Ohne GIS stockt die Digitalisierung"}

Auf der GIS Talk 2018 in Bonn drehte sich zwei Tage lang alles um die Anwendungsmöglichkeiten von Geoinformationssystemen (GIS) und die Potenziale von Location Intelligence. Dabei wurde deutlich: Ohne GIS stockt die Digitalisierung.

Vom 14. bis 15. November kam die GIS-Welt im World Conference Center Bonn zusammen. In mehr als 60 Vorträgen und Tech Tracks zeigten 1.100 GIS-Anwender, IT-Experten und Manager sowie rund 30 Partner und Start-ups, dass Geoinformationssysteme (GIS) eine Schlüsselrolle bei der Digitalisierung spielen und im Hinblick auf globale Herausforderungen wie dem Klimawandel, der Urbanisierung, der Globalisierung oder der Sicherung kritischer Infrastrukturen weiter an Bedeutung gewinnen.

Vom Breitbandausbau über die Energiewende bis hin zur Förderung umweltfreundlicher Mobilitätslösungen oder der Analyse globaler Geschäftsprozesse - bei der Gestaltung des gegenwärtigen und künftigen Zusammenlebens sind Geoinformationssysteme unverzichtbar geworden Denn: Das bloße Sammeln von Daten ebnet noch längst nich den Weg in Richtung Smart Environment, Smart Governance oder Smart City. Stattdessen brauchen Behörden, Unternehmen und Organisationen ein Werkzeug, das Daten in ihren Kontext setzt und komplexe räumliche Zusammenhänge sichtbar macht. Genau das ermöglicht ArcGIS.

Nach einem Grußwort von Bürgermeister Limbach, der auf die Bedeutung von GIS für smarte Städte verwies, sprach der TVModerator Sven Plöger in seiner

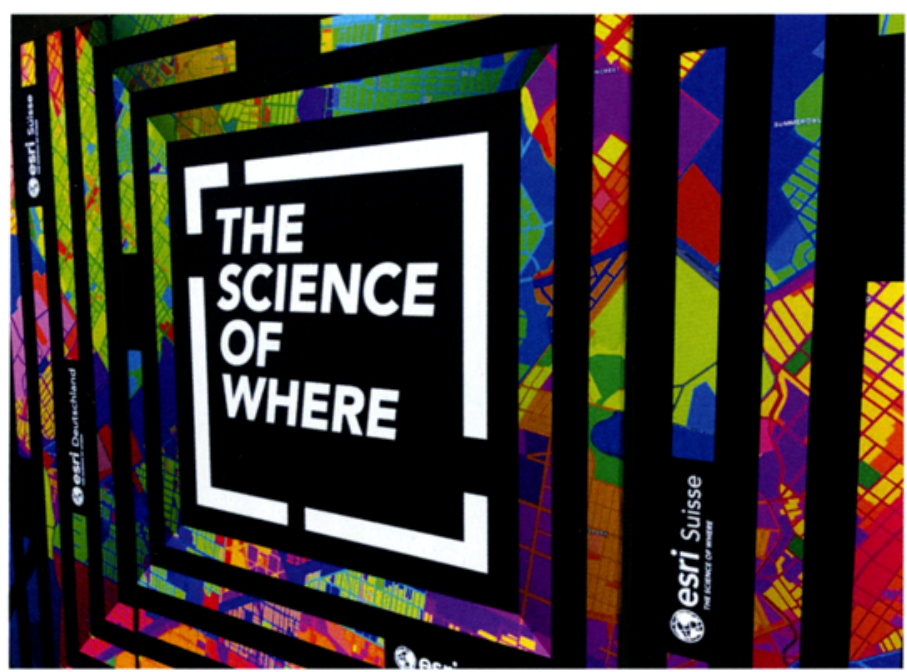

Das Motto der Tagung. Quelle: Esri
Keynote über die Auswirkungen des Klimawandels. Er ordnete zunächst das außergewöhnliche Wettergeschehen des Jahres 2018 ein. Anschließend zeigte er, wie die intelligente Verknüpfung von Geodaten Kommunen und Einsatzkräften hilft, sich präventiv auf extreme Wetterereignisse wie Starkregen und auf das Risiko für Überschwemmungen und Erdrutsche einzustellen. Die Visualisierung von Echtzeitdaten erlaubt es, zu handeln, bevor es zu spät ist.

Nach der Eröffnungs-Plenary lag der Fokus auf den konkreten Anwendungsmöglichkeiten von GIS: In vier verschiedenen Themenforen - Smarte Städte und Regionen, Location Intelligence, Situational Awareness, Infrastructure Intelligence - zeigten mehr als 30 Referentinnen und Referenten, wie sie ArcGIS konkret nutzen und in ihrer jeweiligen Branche von der Lösung profitieren.

Von der Stadtplanung in 3D über das Monitoring von Infrastrukturen und Lieferketten bis hin zu Echtzeit-Lagekarten für Einsatzkräfte - die Themenforen stellten die gesamte Bandbreite der ArcGIS-Plattform dar. Die Vorträge veranschaulichten, dass GIS sowohl für den derzeitigen Ausbau einer leistungsstarken Digital-Infrastruktur als auch im Hinblick auf künftige Big-Dataund Realtime-Analysen größeren Maßstabs essenziell ist.

Während im Themenforum Smarte Städte und Regionen die Themen 3D-Stadtplanung und Sicherheit im urbanen Raum besonders prominent vertreten waren, standen die Optimierung von Lieferketten, die Expansionsplanung und die Standortwahl im Themenforum Location Intelligence im Fokus.

Am zweiten Tag der GIS Talk standen die technischen Grundlagen der ArcGIS-Plattform im Mittelpunkt. Sowohl die technische Plenumsveranstaltung als auch die rund 30 Tech Tracks führten vor Augen, was mit
ArcGIS alles möglich ist - und wie sich die Lösung in bestehende Infrastrukturen integrieren lässt.

Wer die Neuerungen in ArcGIS gleich ausprobieren wollte, kam im Produktzentrum auf seine Kosten. Die Mitarbeiterinnen und Mitarbeiter von Esri standen während der gesamten Konferenz zur Verfügung und lieferten ArcGIS-Knowhow aus erster Hand. Zudem präsentierten EsriPartner, Geschäftspartner und Start-ups unterschiedliche Branchenlösungen, Apps und Dienstleistungen.

Die GIS Talk machte eines mehr als deutlich: Die Zeiten inkompatibler Datensilos sind vorbei. Das Tempo, mit dem die Digitalisierung voranschreitet, verlangt nach offenen Lösungen, die Daten unterschiedlicher Quellen problemlos verknüpfen und in bestehende Prozesse integrieren. Denn nur so lässt sich das Potenzial von Big Data, Realtime oder Machine Learning wirklich nutzen.

Denis Heuring, Esri Deutschland
ICC 2019, Tokio Aufruf zur Einreichung von Fachbeiträgen

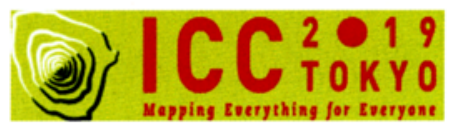

Die 29. Internationale Kartographische Konferenz (ICC2019) findet vom 15.-20. Juli 2019 in Tokio statt. Das Organisationskomitee der ICC2019 lädt alle potenziellen Interessenten ein, Beitragsvorschläge in Form von vollständigen Beiträgen oder Kurzfassungen für Vorträge und Poster einzureichen.

Alle eingereichten Beitragsvorschläge werden von einem internationalen wissenschaftlichen Komitee begutachtet. Alle angenommenen Beiträge werden in einem der folgenden Organe der ICA veröffentlicht: 